\title{
EFICIÊNCIA DE ABSORÇÃO E UTILIZAÇÃO DE FÓSFORO EM PORTA-ENXERTOS CÍTRICOS(1)
}

\author{
Fernando César Bachiega Zambrosi(2), Dirceu Mattos Jr.(3), Pedro \\ Roberto Furlani(2), José Antônio Quaggio(2) \& Rodrigo Marcelli \\ Boaretto $^{(3)}$
}

\begin{abstract}
RESUMO
$O$ porta-enxerto (PE) influencia a nutrição mineral da parte aérea e, consequentemente, a adaptação das árvores cítricas às condições adversas de solo. Considerando que a produtividade dos citros é frequentemente limitada pela baixa disponibilidade de $\mathbf{P}$, foram avaliadas as respostas de crescimento e nutrição fosfatada de plântulas dos PEs limão 'Cravo', citrumelo 'Swingle' e tangerinas 'Cleópatra' e 'Sunki' ao suprimento de 0,0125; 0,05; 0,2; e 0,8 $\mathrm{mmol} \mathrm{L}^{-1}$ de P na solução nutritiva. Após 100 dias de tratamentos, os PEs foram coletados e separados em folhas, ramos e sistema radicular para quantificação de massa seca (MS) e determinação do acúmulo de $P$ nessas partes. Cinco dias antes do término do experimento, folhas e raízes foram amostradas para avaliação da atividade da fosfatase ácida. $O$ suprimento de $P$ na solução nutritiva aumentou a área foliar e a produção de MS da parte aérea e das raízes; os teores foliares e o acúmulo de $\mathrm{P}$ pelos $\mathrm{PEs}$ foram proporcionais à concentração de $\mathrm{P}$ na solução nutritiva. Independentemente do tratamento de $P$, o 'Cravo' apresentou crescimento mais vigoroso, com maior acúmulo de MS e também de $P$, enquanto as tangerinas foram PEs menos vigorosos. A eficiência de absorção de P (EAP) foi incrementada com o suprimento de $P$ na solução nutritiva, e o 'Swingle' foi o $P E$ com menor EAP. $O$ 'Cravo' foi mais eficiente na conversão do $P$ em biomassa nas raízes e, principalmente, na parte aérea. Houve variação na atividade da fosfatase ácida da raiz, indicando que os PEs apresentam distinta capacidade para aproveitamento do $\mathbf{P}$ orgânico do solo. O sistema radicular do 'Cravo' com
\end{abstract}

\footnotetext{
(1) Parte da Tese de Doutorado do primeiro autor apresentada ao Departamento de Biologia Vegetal da Universidade Estadual de Campinas - Unicamp. Recebido para publicação em 9 de junho de 2011 e aprovado em 19 de janeiro de 2012.

(2) Pesquisador, Centro de Solos e Recursos Ambientais, Instituto Agronômico de Campinas, Caixa Postal 28, CEP 13012 -970 Campinas (SP). E-mails: zambrosi@iac.sp.gov.br; pfurlani@conplant.com.br; quaggio@iac.sp.gov.br

(3) Pesquisador, Centro de Citricultura Sylvio Moreira, Instituto Agronômico. Caixa Postal 04, CEP 13490-970 Cordeirópolis (SP). E-mails:ddm@centrodecitricultura.br; boaretto@centrodecitricultura.br
} 
EAP e atividade da fosfatase ácida nas raízes igual ou mais elevada do que a dos demais PEs, crescimento mais vigoroso e conversão mais eficiente do $\mathbf{P}$ em biomassa sugere sua maior adaptação a solos com baixos teores disponíveis de $P$.

Termos de indexação: solução nutritiva, crescimento radicular, nutrição mineral, absorção de fósforo.

\title{
SUMMARY: EFFICIENCY OF PHOSPHORUS UPTAKE AND UTILIZATION IN CITRUS ROOTSTOCKS
}

\begin{abstract}
Citrus rootstocks have a strong influence on shoot mineral nutrition and consequently on the adaptation of citrus trees to adverse soil conditions. Since citrus yield is often limited by low $P$ availability, a greenhouse study was carried out to evaluate the effects of $P$ application in the nutrient solution (0.0125; 0.05; 0.2 and $\left.0.8 \mathrm{mmol} \mathrm{L}^{-1}\right)$ on growth and $P$ nutrition of the citrus rootstocks varieties ('Rangpur' lime, 'Swingle' citrumelo, 'Cleopatra' and 'Sunki' mandarin). After 100 days of $P$ treatments, the rootstocks were harvested and further separated into leaves, stem and root system to determine dry weight and $P$ accumulation. Five days before harvesting the experiment, leaves and roots were sampled to determine acid phosphatase activity. The P supply in the nutrient solution improved leaf area, shoot and root dry weigth. P concentration in the leaves and accumulated $P$ were proportional to the $P$ concentration in the nutrient solution. Regardless of $P$ treatment, 'Rangpur' lime had a more vigorous growth and $P$ accumulation, whereas the mandarin varieties 'Cleopatra' and 'Sunki' had lower shoot and root growth. P uptake efficiency was enhanced by P supply in the nutrient solution, and 'Swingle' had the lowest $P$ uptake efficiency. 'Rangpur' lime was the most efficient rootstock in $P$ utilization for biomass production, especially in the shoot. There was variation in acid phosphatase activity in the roots, suggesting that citrus rootstocks differ in the capacity of using soil organic P. Since 'Rangpur' lime tended to have roots with highest $P$ uptake efficiency, most vigorous growth and highest dry weight per unit of $P$ taken up, as well as acid phosphatase activity in the roots, this might be most the suitable rootstock for low-P soils.
\end{abstract}

Index terms: nutrient solution; root growth; mineral nutrition; phosphorus uptake.

\section{INTRODUÇÃO}

$\mathrm{O}$ uso de porta-enxerto (PE) na cultura dos citros é uma importante ferramenta para melhor adaptação das árvores às condições adversas de solo, como a salinidade (Ruiz et al., 1997, 1999), a toxidez por alumínio (Pereira et al., 2003) e a deficiência de ferro (Pestana et al., 2005). Em solos tropicais, uma das principais limitações ao alcance de produtividades adequadas de frutas cítricas é a disponibilidade insuficiente de $\mathrm{P}$ no solo (Sobral et al., 2000; Quaggio et al., 1998, 2006). Isso pode ser atribuído à baixa concentração de $\mathrm{P}$ na solução do solo $\left(0,1-10 \mu \mathrm{mol} \mathrm{L}^{-1}\right)$ devido à forte interação com a fase sólida (Hinsinger, 2001; Vance et al., 2003), o que restringe a mobilidade do nutriente em direção às raízes e compromete o processo de absorção (Barber et al., 1963; Darrah, 1993). Além disso, ocorre intensa formação de complexos com cátions em solução, que reduz a atividade do íon fosfato e a disponibilidade às plantas (Zambrosi et al., 2008).
A adaptação das plantas à baixa disponibilidade de $\mathrm{P}$ pode ser alcançada mediante utilização mais eficiente do nutriente presente no tecido vegetal para a produção de biomassa (eficiência de utilização) ou por meio de maior capacidade de aquisição de $\mathrm{P}$ do solo (Wang et al., 2010). A variação na eficiência de absorção (quantidade de nutriente absorvida por massa seca de raiz) tem sido relacionada a diferenças no número e comprimento dos pelos radiculares, estabelecimento de associações simbióticas, expressão de transportadores de alta afinidade e alterações na região da rizosfera (Drew, 1975; Liu et al., 1998; Marschner, 1998; Syvertsen \& Graham, 1999; Bates \& Lynch, 2000). O potencial para aproveitamento do P na forma orgânica deve ser um parâmetro que contribua para a tolerância diferencial entre $\mathrm{PEs}$ à baixa disponibilidade de $\mathrm{P}$, uma vez que proporção significativa do $\mathrm{P}$ do solo pode ser encontrada nessa forma (Dalal, 1978; Rubaek \& Sibbesen, 1995). Entretanto, para que o $\mathrm{P}$ orgânico possa ser utilizado como fonte de $\mathrm{P}$, há 
necessidade de solubilização para $\mathrm{P}$ inorgânico por meio da ação da fosfatase ácida. Dessa maneira, a variação na atividade da enzima nas raízes tem sido relacionada à capacidade das plantas para $o$ aproveitamento do $\mathrm{P}$ orgânico no atendimento da demanda pelo nutriente (Asmar, 1997; Richardson et al., 2000; Zhang et al., 2009).

Considerando a função de absorção e translocação de nutrientes para a parte aérea desempenhada pelos PE (Georgiou, 2000), seria importante a caracterização da eficiência de absorção e utilização de P dos PEs mais utilizados na citricultura. Esse conhecimento poderia contribuir para escolha dos materiais genéticos mais adequados à baixa disponibilidade de $\mathrm{P}$ e, também, para explicar as diferenças nas respostas à adubação com $\mathrm{P}$ de árvores sobre distintos PEs cultivados em solos deficientes no nutriente (Mattos Jr. et al., 2006). Sempre que possível, seria vantajoso o emprego dos PEs mais eficientes no uso de $\mathrm{P}$, porque, além de favorecerem o crescimento das árvores em solos com limitações no suprimento de $\mathrm{P}$, contribuiriam para um aproveitamento mais eficiente do nutriente presente no solo e, ou, adicionado por meio de fertilizantes (Baligar et al., 2001; Sinclair \& Vadez, 2002).

O desenvolvimento desta pesquisa foi baseado na hipótese de que os PEs comumente empregados na citricultura diferem na eficiência de absorção e utilização de $\mathrm{P}$, com distintos padrões de respostas à variação na disponibilidade de P. Assim, objetivouse estudar o efeito da concentração de P na solução nutritiva sobre o crescimento e o estado nutricional por P de quatro PEs cítricos.

\section{MATERIAL E MÉTODOS}

O experimento foi realizado em casa de vegetação localizada na área experimental do Instituto Agronômico (IAC), em Campinas-SP, no período compreendido entre setembro e dezembro de 2008. As temperaturas médias diárias variaram entre $31-38{ }^{\circ} \mathrm{C}$ e $14-21{ }^{\circ} \mathrm{C}$, para máxima e mínima, respectivamente. Foram utilizados os portaenxertos (PEs): tangerina 'Cleópatra' (Citrus reshni hort. ex Tanaka), limão 'Cravo' (Citrus limonia Osbeck), tangerina 'Sunki' [Citrus sunki (Hayata) hort. ex Tanaka] e citrumelo 'Swingle' [Citrus paradisi Macfad. cv. Duncan x Poncirus trifoliata (L.) Raf.]. A escolha desses PEs foi feita com base na importância para a citricultura, uma vez que representam aproximadamente $98 \%$ das mudas cítricas produzidas no Estado de São Paulo (Pompeu Jr., 2005).

As plântulas em estádios semelhantes de desenvolvimento (2-3 pares de folhas) e crescidas em tubetes foram adquiridas em viveiro comercial. Após período de aclimatação de sete dias na casa de vegetação, em que se realizou a irrigação com água deionizada, dez plântulas de cada PE foram transferidas para recipientes plásticos com 13,5 L de solução nutritiva diluída a $1 / 4$ e desprovida de $\mathrm{P}$ (Sarruge, 1975; Furlani et al., 1999). Após cinco dias, a solução nutritiva foi renovada por outra com concentração duas vezes maior, seguindo-se o cultivo pelo mesmo período, quando a solução foi substituída por uma com força iônica completa e com os tratamentos de $\mathrm{P}$. O delineamento experimental foi de blocos ao acaso, em esquema de parcela subdividida, com quatro repetições. As parcelas principais foram constituídas pelos tratamentos de $\mathrm{P}\left(\mathrm{C}_{1}=0,0125, \mathrm{C}_{2}\right.$ $=0,05, \mathrm{C}_{3}=0,2 \mathrm{e} \mathrm{C}_{4}=0,8 \mathrm{mmol} \mathrm{L}^{-1}$ de $\mathrm{P}$ na solução nutritiva, fornecido na forma de $\mathrm{KH}_{2} \mathrm{PO}_{4}$ ), enquanto as subparcelas corresponderam aos PEs. Aos 45 dias do transplantio, objetivando maior uniformidade, quatro plantas de cada $\mathrm{PE}$ foram removidas por recipiente, cultivando-se seis plantas até o final do experimento. A solução nutritiva completa apresentava composição equivalente a $9,6 \mathrm{~N}(11 \%$ como $\left.\mathrm{NH}_{4}^{+}\right), 3,6 \mathrm{~K}, 4,0 \mathrm{Ca}, 1,2 \mathrm{Mg}$ e $1,2 \mathrm{mmol} \mathrm{L}^{-1}$ de S; $28 \mathrm{~B}$, $54,0 \mathrm{Fe}, 5,5 \mathrm{Mn}, 2,1 \mathrm{Zn}, 1,1 \mathrm{Cu}$ e $0,01 \mu \mathrm{mol} \mathrm{L}^{-1}$ de Mo (Sarruge, 1975; Furlani et al., 1999) e foi renovada a cada dez dias, mantendo-se o volume dos recipientes constante com adição de água deionizada. $\mathrm{O} \mathrm{pH}$ foi ajustado para 5,5-5,7, e o fornecimento contínuo de oxigênio foi feito por meio de compressor de ar.

A atividade da fosfatase ácida in vivo nas folhas (AFAF) foi realizada aos 95 dias após o início dos tratamentos, segundo os procedimentos descritos por Besford (1980) e Elliott \& Lauchli (1986). Foram retirados seis discos com $5 \mathrm{~mm}$ de diâmetro (massa fresca total de 0,5-0,6 g) do limbo de folhas do terço médio inferior, e colocados para incubar em tubos de ensaio contendo $1 \mathrm{~mL}$ de acetato de sódio $50 \mathrm{mmol} \mathrm{L}^{-1}$ a pH 5,0 e $1 \mathrm{~mL}$ de $11,0 \mathrm{mmol} \mathrm{L}^{-1}$ de paranitrofenilfosfato. Os tubos permaneceram durante 15 min em banho termostático a $30{ }^{\circ} \mathrm{C}$, quando alíquotas de $1 \mathrm{~mL}$ foram transferidas para tubos com $2 \mathrm{~mL}$ de $\mathrm{NaOH} 1 \mathrm{~mol} \mathrm{~L}^{-1}$, de modo que a reação fosse paralisada. As leituras foram imediatamente realizadas em espectrofotômetro a $410 \mathrm{~nm}$, confrontando-se com uma curva-padrão de paranitrofenol. Os resultados foram expressos em $\mu \mathrm{mol}$ de paranitrofenol por g de material vegetal fresco por min, correspondendo à atividade extracelular da enzima (Fernandez \& Ascencio, 1994). Para determinação da atividade da enzima nas raízes (AFAR), seguiu-se o mesmo procedimento adotado para as folhas, utilizando-se fragmentos de aproximadamente 0,5 g da região apical das raízes secundárias (Speir \& Cowlling, 1991; Fernandez \& Ascencio, 1994) dos PEs cultivados em 0,0125 e $0,8 \mathrm{mmol} \mathrm{L}^{-1}$ de $\mathrm{P}$.

Após 100 dias do início dos tratamentos, os PEs foram coletados e separados em folhas, ramos e 
sistema radicular. O correspondente a 20 discos foliares de $5 \mathrm{~mm}$ de diâmetro foi retirado para a estimativa da área foliar ( $\mathrm{AF}, \mathrm{cm}^{2} /$ planta). Os discos foram secos e pesados, e a AF foi calculada por meio da relação direta entre a MS dos discos foliares e a MS total de folhas. As diferentes partes foram lavadas em água deionizada e colocadas para secar em estufa de circulação forçada de ar a $65^{\circ} \mathrm{C}$ por $72 \mathrm{~h}$, para quantificação da MS (g/planta). A análise do teor de $\mathrm{P}\left(\mathrm{g} \mathrm{kg}^{-1}\right)$ no tecido vegetal foi feita segundo Bataglia et al. (1983), com a quantificação da concentração de $\mathrm{P}$ nos extratos baseada na formação do complexo P-molibdato (Malavolta et al., 1997). O P acumulado pelos PEs (mg/planta) foi obtido a partir do produto entre o teor de $\mathrm{P}$ nas diferentes partes e o acúmulo de MS. A partir desses resultados, calcularam-se: i) eficiência de absorção de $\mathrm{P}\left(\mathrm{EAP}, \mathrm{mg} \mathrm{g}^{-1}\right)=$ (conteúdo total de $\mathrm{P}$ no $\left.\mathrm{PE}, \mathrm{mg}\right) /$ (MS do sistema radicular, g) (Swiader et al., 1994); e ii) eficiência de utilização de $\mathrm{P}$ na $\mathrm{PA}$ e nas raízes $\left(\right.$ EUP, $\left.\mathrm{g}^{2} \mathrm{mg}\right)=(\text { MS da PA ou raízes, } \mathrm{g})^{2} /$ (conteúdo de P na PA ou nas raízes, mg) (Siddiqi \& Glass, 1981).

Os resultados foram submetidos à análise de variância, considerando-se o esquema de parcelas subdivididas. No caso de interação significativa entre tratamentos de P e PEs, o efeito dos tratamentos para cada PE foi determinado por análise de regressão (ajuste linear ou quadrático) ou comparado pelo teste de Duncan a 0,05, que também foi usado na comparação entre os PEs. Análise de correlação também foi estabelecida entre variáveis previamente selecionadas.

\section{RESULTADOS}

\section{Crescimento e estado nutricional por fósforo}

A área foliar (AF) e o acúmulo de MS na PA e nas raízes dos PEs aumentaram com a adição de $\mathrm{P}$ na solução nutritiva; contudo, a resposta foi diferenciada, como indicado pela interação significativa $(p<0,05)$ entre tratamentos de P e PEs (Figura 1a,c). Por exemplo, a AF da 'Sunki' respondeu de forma linear, variando de $58,3 \mathrm{~cm}^{2}$ / planta no $\mathrm{C}_{1}$ a $117,6 \mathrm{~cm}^{2} /$ planta no $\mathrm{C}_{4}$ (Figura 1a). Para os demais PEs, essa variável foi ajustada ao modelo quadrático, com AF máxima de 58,8, 365,2 e 182,0 cm²/planta para 'Cleópatra', 'Cravo' e 'Swingle', respectivamente, o que requereu $0,49-0,57 \mathrm{mmol} \mathrm{L}^{-1}$ de P na solução nutritiva.

A MS da PA do 'Cravo' e do 'Swingle' também respondeu de forma quadrática ao $\mathrm{P}$ (Figura 1b), o que permitiu a definição de $0,50-0,52 \mathrm{mmol} \mathrm{L}^{-1}$ de $\mathrm{P}$ como a concentração requerida para o máximo crescimento estimado (8,2 e 2,9 g/planta para o 'Cravo' e 'Swingle', respectivamente). Por outro lado, a MS da PA de 'Cleópatra' e 'Sunki' ajustou-se ao modelo linear (Figura 1b), sendo este último PE, com base nos coeficientes angulares das equações, mais responsivo ao $\mathrm{P}$. Respostas lineares também foram observadas para MS das raízes, sendo o 'Cravo' e 'Cleópatra' aqueles com crescimento radicular mais e menos responsivo ao nutriente, respectivamente (Figura 1c). $\mathrm{O}$ ganho de $\mathrm{MS}$ das raízes entre $\mathrm{C}_{1}$ e $\mathrm{C}_{4}$ estimado pelas equações de regressão correspondeu a 0,$09 ; 0,45 ; 0,25 ;$ e $0,17 \mathrm{~g} /$ planta para 'Cleópatra', 'Cravo', 'Sunki' e 'Swingle', respectivamente.

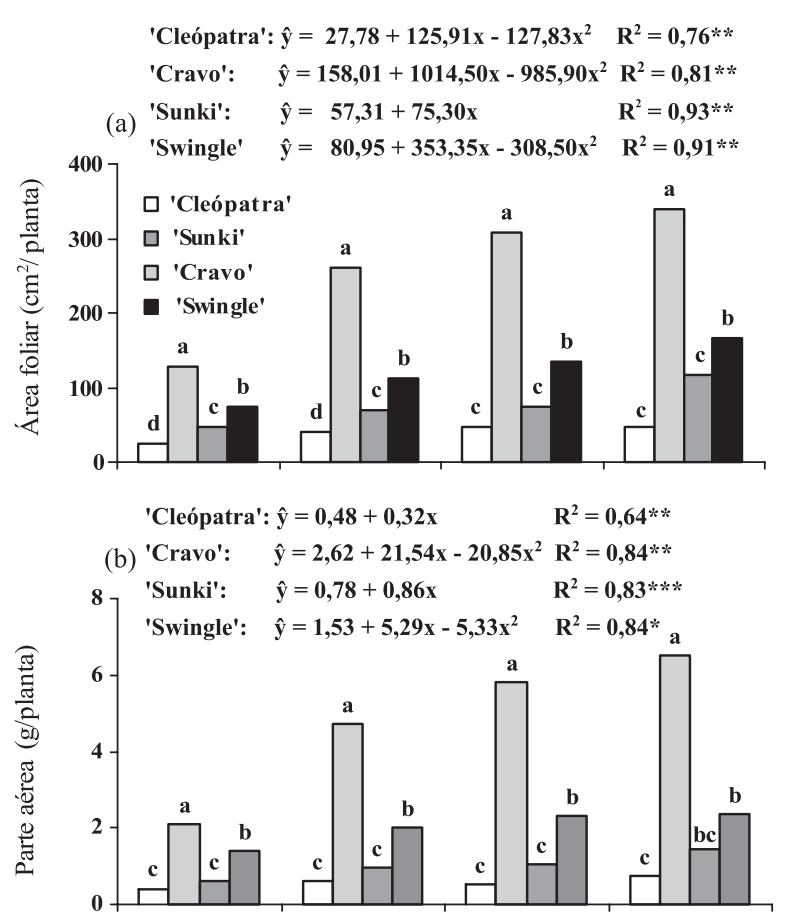

(c)

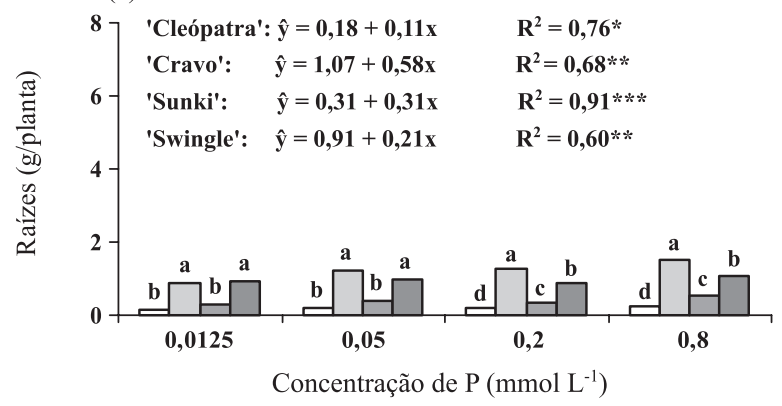

Figura 1. Área foliar (a) e crescimento da parte aérea (b) e das raízes (c) de quatro portaenxertos cítricos submetidos a concentrações de $\mathbf{P}$ na solução nutritiva. Comparação entre porta-enxertos: colunas com letras iguais dentro do mesmo tratamento de $\mathrm{P}$ não diferem pelo teste de Duncan a 0,05 . Comparação entre tratamentos de P: o efeito da concentração de $P$ foi ajustado a modelos de regressão para cada porta-enxerto, em que $\hat{y}$ = área foliar, massa seca da parte aérea ou massa seca das raízes; $\mathbf{e} x$ $=$ mmol $\mathrm{L}^{-1}$ de $\mathrm{P}$ na solução nutritiva. ${ }^{*} \mathrm{p}<0,05$; $* * \mathrm{p}<0,01 ; * * * \mathrm{p}<0,001 .(\mathrm{n}=16)$. 
Independentemente do tratamento, o 'Cravo' apresentou maior AF e MS da PA, sendo seguido pelo 'Swingle' e, por fim, pelas tangerinas (Figura 1a,b). Por exemplo, no tratamento $\mathrm{C}_{1}$, a diferença da AF e MS da PA do 'Cravo' em relação aos demais correspondeu a $1,8-5,4$ e $1,5-5,6$ vezes, respectivamente. Nos tratamentos $\mathrm{C}_{1}$ e $\mathrm{C}_{2}$ não houve variação no crescimento radicular entre as tangerinas, e do 'Cravo' em relação ao 'Swingle' (Figura 1c). Entretanto, em $\mathrm{C}_{3}$ e $\mathrm{C}_{4}$, a seguinte ordem de MS das raízes foi observada: 'Cravo' > 'Swingle' > 'Sunki' > 'Cleópatra'.

A interação significativa $(p<0,05)$ entre tratamentos de $\mathrm{P}$ e PEs foi encontrada para teores foliares e acúmulo total de $\mathrm{P}$ na planta, sugerindo resposta distinta da nutrição fosfatada entre os PEs ao suprimento do nutriente na solução nutritiva (Figura 2a,b). A concentração de $\mathrm{P}$ requerida para os máximos teores correspondeu a 0,50-0,60 $\mathrm{mmol} \mathrm{L}^{-1}$, as quais resultaram em 2,$8 ; 2,9 ; 3,1 ;$ e $2,4 \mathrm{~g} \mathrm{~kg}^{-1}$

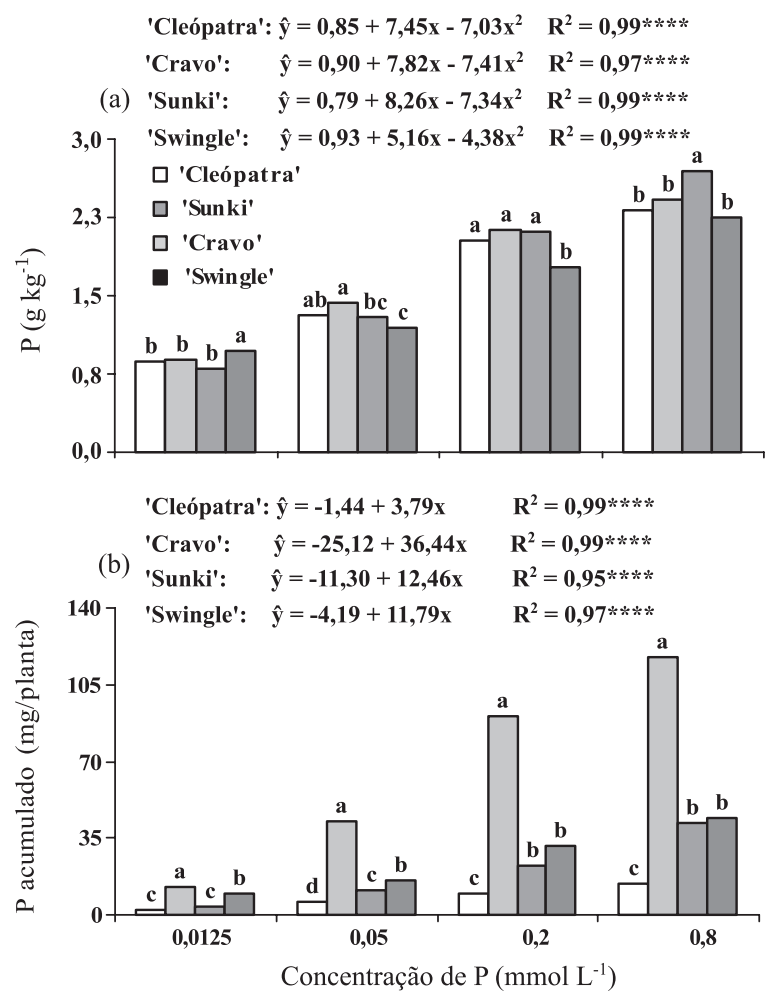

Figura 2. Teor de $\mathbf{P}$ nas folhas (a) e P acumulado (b) por quatro porta-enxertos cítricos submetidos a concentrações de $\mathbf{P}$ na solução nutritiva. Comparação entre porta-enxertos: colunas com letras iguais dentro do mesmo tratamento de $\mathbf{P}$ não diferem pelo teste de Duncan a 0,05 . Comparação entre tratamentos de P: o efeito da concentração de $\mathbf{P}$ foi ajustado a modelos de regressão para cada porta-enxerto, em que $\hat{y}=$ teor de $\mathbf{P}$ ou $P$ acumulado; $\mathrm{e} x=\mathrm{mmol} \mathrm{L}^{-1} \mathrm{de}$ $\mathrm{P}$ na solução nutritiva. ****p $<0,0001 .(\mathrm{n}=16)$ de P nas folhas de 'Cleópatra', 'Cravo', 'Sunki' e 'Swingle', respectivamente. No tratamento $\mathrm{C}_{1}$, o 'Swingle' apresentou o teor mais elevado de $\mathrm{P}$ nas folhas, enquanto resultado oposto foi obtido em $\mathrm{C}_{3}$ (Figura 2a). A 'Sunki' teve os maiores teores quando cultivada na maior disponibilidade de $\mathrm{P}$ na solução nutritiva $\left(0,8 \mathrm{mmol} \mathrm{L}^{-1}\right)$, porém sem variação entre os outros PEs.

$\mathrm{O}$ acúmulo de $\mathrm{P}$ pelos $\mathrm{PEs}$ foi proporcional ao fornecimento do nutriente, com 'Cleópatra' e 'Cravo' sendo o menos e o mais responsivo, respectivamente (Figura 2b). O 'Cravo' acumulou mais $\mathrm{P}$ em todos os tratamentos, sendo seguido pelo 'Swingle' e 'Sunki', os quais não diferiram em $\mathrm{C}_{3}$ e $\mathrm{C}_{4}$ (Figura 2b). $\mathrm{O}$ crescimento dos $\mathrm{PEs}$ foi correlacionado com os teores foliares de P (Figura 3) e, com base nas equações, determinou-se que os teores correspondentes ao maior acúmulo de MS foram de 2,0; 2,4; 3,7; e 2,3 g kg-1 de P para 'Cleópatra', 'Cravo', 'Sunki' e 'Swingle', respectivamente.

\section{Eficiência de absorção e utilização de fósforo}

Foi observada interação significativa $(\mathrm{p}<0,05)$ entre tratamentos de $\mathrm{P}$ e PEs para a eficiência de absorção (EAP) e de utilização de P (EUP) para a produção de biomassa na PA e nas raízes (Quadro 1). $\mathrm{O}$ suprimento de $\mathrm{P}$ aumentou a EAP: em $\mathrm{C}_{4}$, a EAP foi 4,$3 ; 5,2 ; 5,6$; e 4,2 vezes mais elevada em relação ao $\mathrm{C}_{1}$, para 'Cleópatra', 'Cravo', 'Sunki' e 'Swingle', respectivamente. O 'Swingle' foi consistentemente o $\mathrm{PE}$ menos eficiente para absorção de $\mathrm{P}$, ao passo que nos tratamentos $\mathrm{C}_{2}$ e $\mathrm{C}_{3}$ o 'Cravo' apresentou EAP 27-144\% mais elevada que a dos demais.

Exceto nos tratamentos $\mathrm{C}_{1}$ e $\mathrm{C}_{2}$, em que a EUP nas raízes foi semelhante à do 'Swingle', o 'Cravo' teve a mais elevada EUP da PA e das raízes entre os PEs (Quadro 1). O 'Swingle' foi o segundo PE mais eficiente na utilização do P, enquanto 'Cleópatra' e 'Sunki' tiveram os menores valores. Por exemplo, ao longo dos tratamentos de P, a EUP na PA e nas raízes do 'Cravo', em relação a 'Cleópatra', foi 4,8-10,4 e $8,0-9,8$ vezes mais elevada, respectivamente. Para todos os PEs, a EUP na PA e nas raízes foi reduzida mediante a adição de $\mathrm{P}$ na solução nutritiva; na comparação entre $\mathrm{C}_{1}$ e $\mathrm{C}_{4}$, a EUP na $\mathrm{PA}$ e nas raízes diminuiu em 32-42 e 60-70\%, respectivamente (Quadro 1).

\section{Atividade da fosfatase ácida}

A atividade da fosfatase ácida nas folhas (AFAF) foi influenciada pelos PEs e pela concentração de P na solução nutritiva, sem, contudo, ocorrer interação significativa $(p>0,05)$ (Quadro 1). Na média dos PEs, a AFAF foi $60 \%$ mais elevada no $\mathrm{C}_{1}$ em relação aos demais tratamentos, os quais não diferiram entre si. Na comparação entre os PEs, para a média 
dos tratamentos de $\mathrm{P}$, a AFAF foi menor para o 'Swingle' quando comparado a 'Cleópatra' e 'Cravo', que por sua vez tiveram AFAF semelhante à de 'Sunki' (Quadro 1).
Diferentemente do observado com a AFAF, para a atividade da enzima nas raízes (AFAR) ocorreu interação significativa $(\mathrm{p}<0,05)$ entre tratamentos de $\mathrm{P}$ e PEs (Quadro 2). Exceto para o 'Swingle',
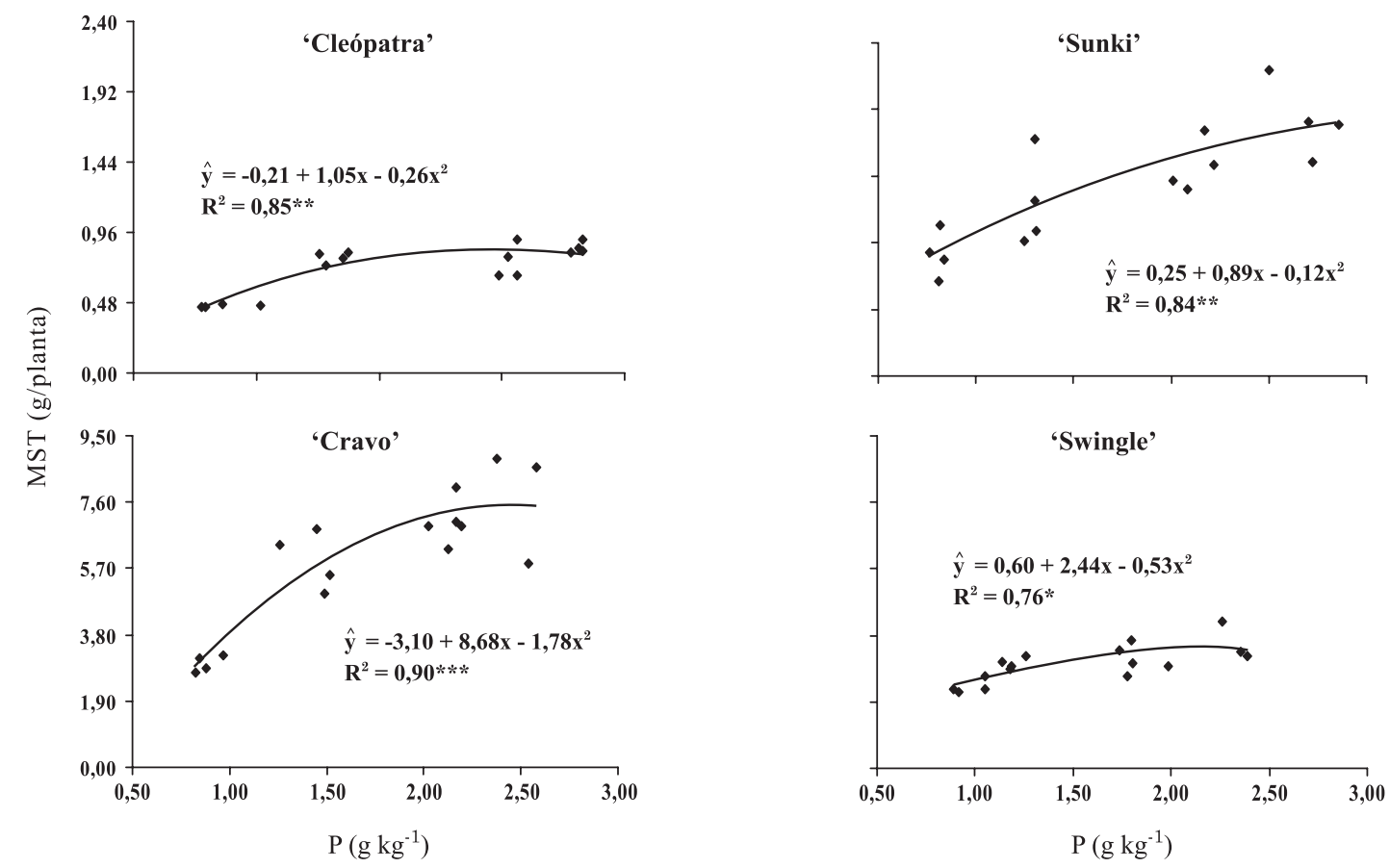

Figura 3. Massa seca total (MST) de quatro porta-enxertos cítricos cultivados em solução nutritiva, em função do teor de P nas folhas. *p $<0,05 ; * * \mathrm{p}<0,01$; ***p $<0,001 .(\mathrm{n}=16)$.

Quadro 1. Eficiência de absorção de fósforo (EAP), eficiência de utilização de fósforo (EUP) na parte aérea e nas raízes e atividade da fosfatase ácida nas folhas (AFAF) de quatro porta-enxertos cítricos cultivados em diferentes concentrações de $\mathbf{P}$ na solução nutritiva

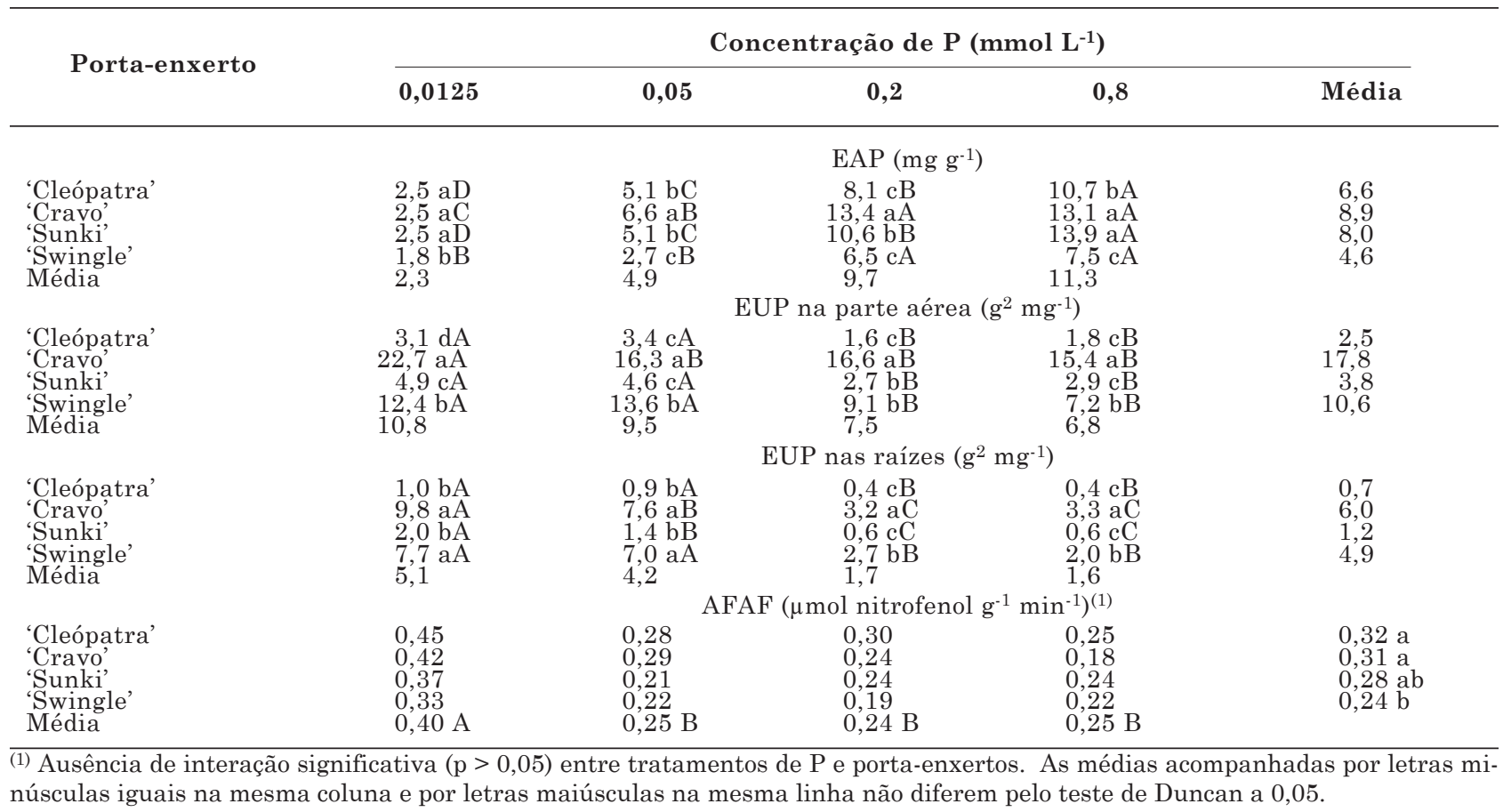


a AFAR no $\mathrm{C}_{1}$ foi maior do que no tratamento $\mathrm{C}_{4}$, com acréscimos de 84, 125 e $153 \%$ para 'Cleópatra', 'Cravo' e 'Sunki', respectivamente. $\mathrm{No}_{4}$ não houve diferença entre os PEs, enquanto para $\mathrm{C}_{1}$ o 'Cravo' teve AFAR mais elevada do que a de 'Cleópatra' e 'Swingle', porém não diferiu da de 'Sunki'.

Quadro 2. Atividade da fosfatase ácida nas raízes (AFAR) de quatro porta-enxertos cítricos cultivados em duas concentrações de fósforo na solução nutritiva

\begin{tabular}{lccc}
\hline $\begin{array}{c}\text { Porta- } \\
\text { enxerto }\end{array}$ & \multicolumn{2}{c}{ Concentração de $\mathbf{P}\left(\mathbf{m m o l ~ L}^{-\mathbf{1}}\right)$} & Média \\
\cline { 2 - 3 } & $\mathbf{0 , 0 1 2 5}$ & $\mathbf{0 , 8}$ & \\
\hline & AFAR $\left(\mu \mathrm{mol}\right.$ nitrofenol g-1 $\left.\mathrm{min}^{-1}\right)$ & \\
'Cleópatra' & $0,35 \mathrm{bA}$ & $0,19 \mathrm{aB}$ & 0,27 \\
'Cravo' & $0,54 \mathrm{aA}$ & $0,24 \mathrm{aB}$ & 0,39 \\
'Sunki' & $0,43 \mathrm{abA}$ & $0,17 \mathrm{aB}$ & 0,30 \\
'Swingle' & $0,21 \mathrm{cA}$ & $0,24 \mathrm{aA}$ & 0,23 \\
Média & 0,38 & 0,21 & \\
& &
\end{tabular}

As médias acompanhadas por letras minúsculas iguais na mesma coluna e por letras maiúsculas na mesma linha não diferem pelo teste de Duncan a 0,05.

\section{DISCUSSÃO}

Os resultados obtidos confirmaram a hipótese do trabalho de que os PEs cítricos difeririam na EAP e EUP para a produção de biomassa da PA e das raízes (Quadro 1). O 'Swingle' foi em todos os tratamentos de $\mathrm{P}$ o $\mathrm{PE}$ com sistema radicular menos eficiente na absorção de $\mathrm{P}$ (quantidade de nutriente absorvido por MS de raiz), ao passo que em $\mathrm{C}_{2}$ e $\mathrm{C}_{3}$ o 'Cravo' foi o mais eficiente. Diferenças na morfologia e fisiologia radicular que alteram as características de absorção de nutrientes têm sido determinadas entre PEs (Eissenstat \& Achor, 1999; Sorgoná et al., 2005; Zambrosi et al., 2011), o que deve alterar consequentemente a EAP. O crescimento radicular distinto entre os PEs (Figura 1c) contribuiu para variações nas quantidades absorvidas de $\mathrm{P}$, visto que foi estabelecida correlação positiva entre o acúmulo de $\mathrm{P}$ com a MS de raízes $(\mathrm{r}=0,75 ; \mathrm{p}<0,0001 ; \mathrm{n}=$ 64). Dessa maneira, considerando que o 'Cravo' teve EAP igual ou mais elevada do que a dos demais PEs ao longo de contrastantes disponibilidades de $\mathrm{P}$, e também sistema radicular com crescimento mais vigoroso e responsivo ao $\mathrm{P}$, é provável que árvores sobre esse $\mathrm{PE}$ tenham maior potencial para aquisição de P. Por exemplo, foi observado em condições de solução nutritiva, que laranjeira 'Pêra' enxertada sobre 'Cravo' apresentou maior capacidade de absorção de $\mathrm{P}$ e atendimento da demanda da PA em comparação àquelas sobre 'Cleópatra' (Zambrosi et al., 2012). Esses resultados são condizentes com os obtidos em campo, no qual árvores sobre 'Cravo' cultivadas em solo com baixa disponibilidade de $\mathrm{P}$ tiveram maior produção de frutos do que aquelas sobre 'Cleópatra' (Mattos Jr. et al., 2006), as quais requerem doses mais elevadas de $\mathrm{P}$ na implantação dos pomares (Quaggio et al., 2005).

$\mathrm{O}$ suprimento de $\mathrm{P}$ na solução nutritiva resultou em aumento da EAP dos PEs (Quadro 1), sugerindo que concentrações mais elevadas do nutriente no meio de cultivo favoreceram um funcionamento mais eficiente das raízes no processo de absorção, conforme já observado para outras culturas (Gourley et al., 1993). O aumento da EAP foi também provavelmente relacionado ao efeito positivo do suprimento de $\mathrm{P}$ sobre o acúmulo do nutriente pela PA, como indicado por correlações positivas entre essas duas variáveis ('Cleópatra': $\mathrm{r}=0,84$, $\mathrm{p}<0,0001, \mathrm{n}=16$; 'Cravo': $\mathrm{r}=0,96, \mathrm{p}<0,0001, \mathrm{n}=$ 16; 'Sunki': $r=0,90, p<0,0001, n=16$; e 'Swingle': $r=0,84, p<0,0001, n=16)$. Isso era de certo modo esperado, uma vez que a demanda da PA atua como o principal fator regulador nas quantidades absorvidas de nutrientes (Marschner, 1995). Além disso, a EAP foi calculada pela relação entre o P acumulado pelas plantas e MS das raízes, a qual ocupou menor proporção do acúmulo total de MS pelos PEs nas concentrações mais elevadas de P, como indicado pela queda da relação raiz/PA (dados não mostrados).

A EUP na PA e raízes decresceu com o suprimento de $\mathrm{P}$, especialmente nos tratamentos $\mathrm{C}_{3}$ e $\mathrm{C}_{4}$ (Quadro 1), confirmando a redução da eficiência de utilização com o aumento da disponibilidade de nutrientes (Elliot \& White, 1994; Sanginga at al., 2000), desde que os ganhos de biomassa não ocorrem na mesma magnitude em que os nutrientes são absorvidos e acumulados no tecido vegetal (Marschner, 1995). Em condições de disponibilidade mais elevada ocorre o armazenamento de $\mathrm{P}$ no vacúolo na forma inorgânica (Hart \& Jessop, 1983), o qual contribui para elevar o teor do nutriente na planta, porém sem participar diretamente de reações metabólicas e, ou, formação de estruturas (P orgânico), ao passo que, para plantas cultivadas em baixa disponibilidade, a fração presente no vacúolo contribui em menor proporção para o $\mathrm{P}$ total (Furtini Neto et al., 1998; Martinez et al., 2005). Em condições de deficiência por P, a EUP é aumentada como resultado de alterações na atividade de enzimas na rota da glicólise, que passam a operar em vias alternativas no catabolismo de carboidratos durante a deficiência, contribuindo para uso mais eficiente do $\mathrm{P}$ por meio de reciclagem, redução de consumo e utilização de outras frações do nutriente (Theodoru \& Plaxton, 1993; Shenoy \& Kalagudi, 2005).

A EUP distinta entre os PEs indicou a variabilidade na conversão do $\mathrm{P}$ absorvido em crescimento, o que está de acordo com resultados 
encontrados para outras culturas (Fageria et al., 1988; Morais et al., 1990; Moura et al., 2001; Furlani et al., 2002; Pinto et al., 2011). A EUP na PA ou nas raízes foi diretamente correlacionada com MS da PA $(r=0,78, p<0,0001, \mathrm{n}=64)$ e MS das raízes $(\mathrm{r}=0,61, \mathrm{p}<0,0001, \mathrm{n}=64)$, respectivamente. Essa associação positiva entre EUP e crescimento dos PEs pode ser atribuída à variação da AF (Figura 1a), à condutividade hidráulica das raízes (Syvertsen \& Graham, 1985) e à atividade fotossintética (Zambrosi et al., 2011), as quais alteram marcadamente os ganhos de MS pelas plantas e, consequentemente, a eficiência de utilização dos nutrientes absorvidos em produção de biomassa. As diferenças no desenvolvimento da $\mathrm{AF}$ e no acúmulo de MS na PA e raiz para os mesmos tratamentos de $\mathrm{P}$ (Figura 1ac) suportam a variação de vigor entre PEs cítricos (Carvalho, 1994; Esposti \& Siqueira, 2004), com maiores diferenças observadas do 'Cravo' em relação às tangerinas. A EUP das raízes seguiu o mesmo padrão da PA, com 'Cleópatra' e 'Sunki' sendo os PEs menos eficientes (Quadro 1). A utilização mais eficiente pelo 'Cravo' e 'Swingle' do P para a construção de biomassa radicular, especialmente em condições de deficiência do nutriente, contribuiria para maior exploração do solo por unidade de $\mathrm{P}$ contido nas raízes, resultando, consequentemente, em maior aquisição e quantidade de $\mathrm{P}$ disponível para translocação e utilização na PA.

Os resultados do presente trabalho indicaram que os PEs cítricos diferem nas respostas de crescimento à variação da disponibilidade de $\mathrm{P}$ no ambiente radicular, conforme suportado pela interação significativa $(p<0,05)$ entre tratamentos de P e PEs para AF, MS da PA e das raízes (Figura 1a-c). Embora essas variáveis tenham respondido de forma positiva à adição de $\mathrm{P}$ na solução nutritiva, o padrão de resposta foi distinto entre os PEs, com destaque para o 'Cravo', que foi muito mais responsivo ao $\mathrm{P}$ (ganho absoluto de AF ou MS para cada mmol de $\mathrm{P}$ adicionado na solução nutritiva) do que os demais PEs. Em condições de deficiência de P, o menor crescimento das plantas é atribuído à queda de $\mathrm{AF}$ disponível para interceptação de radiação fotossinteticamente ativa, o que ocasiona decréscimo na quantidade de carboidratos produzidos e disponíveis para atender à demanda de crescimento (Mollier \& Pellerin, 1999). Isto foi suportado pela correlação positivada da AF com MS da PA ( $\mathrm{r}=$ $0,98, \mathrm{p}<0,0001, \mathrm{n}=64)$ e MS das raízes $(\mathrm{r}=0,74$, $\mathrm{p}<0,0001, \mathrm{n}=64$ ) dos PEs ao final do experimento. A redução da $\mathrm{AF}$ em baixa disponibilidade de $\mathrm{P}$ é atribuída ao menor aparecimento de folhas, como ocorrido no presente trabalho (dados não mostrados), e a prejuízos na elongação de folhas individuais (Lynch et al., 1991), devido à limitação na expansão de células da epiderme com a deficiência de fosfato (Fredden et al., 1989) e no número de divisões celulares (Chiera et al., 2002). Além da redução absoluta da $\mathrm{AF}$, o ganho de biomassa por unidade de $\mathrm{AF}$ é menor em plantas deficientes em $\mathrm{P}$, em razão da maior demanda respiratória de tecidos heterotróficos, ocorrendo maior degradação do carbono fixado pela fotossíntese na respiração radicular (Nielsen et al., 2001). Isso ocorre porque, nos PEs deficientes, a partição de $\mathrm{C}$ é alterada, ocorrendo maior alocação relativa de biomassa no sistema radicular e consequente aumento da relação raiz/PA (Syvertsen \& Graham, 1999), uma vez que há distribuição preferencial de fotoassimilados para as raízes em detrimento da PA (Rao \& Terry, 1995). $\mathrm{O}$ efeito do estado nutricional de $\mathrm{P}$ em determinar a partição de biomassa entre raiz e PA foi confirmado por meio da correlação negativa entre o teor de $\mathrm{P}$ nas folhas e R/PA $(r=-0,31, p=0,01, n=64)$. Além disso, a relação AF/MS de raízes foi influenciada pelo suprimento de $\mathrm{P}$, obtendo-se menos unidade de AF disponível para sustentar a demanda de cada unidade de MS do sistema radicular das plantas deficientes (dados não mostrados).

Os teores foliares e a absorção de $\mathrm{P}$ pelos $\mathrm{PEs}$ foram incrementados mediante a adição desse nutriente na solução nutritiva (Figura 2a-b), indicando que os tratamentos utilizados resultaram em PE com estado nutricional distinto, tendo-se plantas deficientes e suficientes em P (Bataglia et al., 2008). Além disso, os teores foliares de $\mathrm{P}$ foram positivamente correlacionados com o crescimento dos PEs, demonstrando a limitação imposta pela deficiência do nutriente aos ganhos de MS (Figura 3). Com base nesses modelos, verificouse que houve diferenças no crescimento entre os PEs em função de teores semelhantes de P. Por exemplo, na comparação entre 'Cleópatra' e 'Cravo', o máximo acúmulo de MS do primeiro correspondeu a $0,85 \mathrm{~g} /$ planta para um teor de $2,0 \mathrm{~g} \mathrm{~kg}^{-1}$ de $\mathrm{P}$, enquanto o 'Cravo' apresentou crescimento máximo 8,8 vezes mais elevado $(7,48 \mathrm{~g} /$ planta) para um teor do nutriente somente 1,2 vez maior $\left(2,4 \mathrm{~g} \mathrm{~kg}^{-1}\right)$ (Figura 3), refletindo pronunciadas diferenças na capacidade de produção de MS mediante a manutenção de teores semelhantes de $\mathrm{P}$ nas folhas. Ainda, as distintas quantidades acumuladas de $\mathrm{P}$ entre os PEs (Figura 2b) indicaram variação no requerimento por $\mathrm{P}$ e na capacidade de absorção do nutriente, a qual deve estar relacionada com o vigor de crescimento de cada material genético. Isso foi confirmado pelo fato de que o crescimento foi bem correlacionado com o acúmulo de $\mathrm{P}$ nas plantas $(\mathrm{r}$ $=0,89, \mathrm{p}<0,0001, \mathrm{n}=64)$. Ao contrário do teor de $\mathrm{P}$, a atividade da fosfatase ácida nas folhas de cada PEs não respondeu de forma distinta ao suprimento de $\mathrm{P}$, conforme indicado pela ausência de interação significativa $(p>0,05)$ entre tratamentos de $\mathrm{P}$ e PEs (Quadro 1). Na média dos tratamentos de $\mathrm{P}$, diferença na AFAF foi observada na comparação do 'Swingle' com 'Cleópatra' e 'Cravo', que deve estar relacionada à variação no teor da proteína e, 
ou, atividade específica de diferentes isoenzimas (Fernandez \& Ascencio, 1994; Lim et al., 2003). A AFAF foi negativamente correlacionada com os teores foliares de $\mathrm{P}(\mathrm{r}=-0,47, \mathrm{p}<0,0001, \mathrm{n}=64)$, conforme encontrado para outras culturas (Besford, 1979; O’Connell \& Grove, 1985; López-Cantero et al., 1998; Yan et al., 2001), o que justificaria o emprego da AFAF para avaliação do estado nutricional. Entretanto, mesmo com incrementos significativos nos teores foliares e na quantidade acumulada de $\mathrm{P}$ entre os tratamentos $\mathrm{C}_{2}$ e $\mathrm{C}_{4}$ (Figura 2a,b), não houve diminuição significativa da AFAF (Quadro 1). A AFA foi mais sensível em detectar variação no estado nutricional quando a deficiência por $\mathrm{P}$ foi mais severa (tratamento com $0,0125 \mathrm{mmol} \mathrm{L}^{-1}$ de P), corroborando estudos de Elliot \& Läuchli (1986), em que a AFAF não foi hábil para refletir variação no estado nutricional em condições moderadas de deficiência, mas somente em plantas severamente deficientes.

Uma das respostas que condicionam maior adaptação à baixa disponibilidade de $\mathrm{P}$ e contribuem para aumentar a EAP é a secreção de fosfatase ácida pelo sistema radicular (Gilbert et al., 1999; Yun \& Kaepler, 2001; Nanamori et al., 2004;), pois promove a quebra de ésteres de fosfato e libera $\mathrm{P}$ inorgânico na solução do solo, disponibilizando-o para absorção pelas raízes (Helal, 1990; Quisel et al., 1996; Tomscha et al., 2004). Os resultados deste trabalho indicaram que, em condições de baixa disponibilidade de $\mathrm{P}$, o 'Cravo', mesmo que sua AFAR não tenha diferido da de 'Sunki' (Quadro 2), seria mais eficiente do que os demais PEs no aproveitamento da fração de $\mathrm{P}$ orgânico. Isso seria importante para adaptação em solos com baixa disponibilidade de $\mathrm{P}$, uma vez que o P orgânico pode ocupar entre 30 e 80 \% do total do nutriente encontrado no solo em áreas cultivadas (Bieleski, 1973). O estado nutricional por P dos PEs alterou diretamente o nível de atividade da enzima nas raízes, como confirmado pelas correlações negativas da AFAR com o teor de $\mathrm{P}$ nas folhas $(\mathrm{r}$ $=-0,66, \mathrm{p}<0,0001, \mathrm{n}=32)$ e nas raízes $(\mathrm{r}=-0,63$, $\mathrm{p}=0,0001, \mathrm{n}=32$ ). Isso ocorre porque a atividade é mais alta quando concentrações deficientes na PA são atingidas (Furlani et al., 1984) ou quando há decréscimos no teor de $\mathrm{P}$ nas raízes (Speir \& Cowling, 1991), levando à maior síntese da proteína e, ou, à ativação daquela preexistente (Duff et al., 1991; Coelho, 2002). Além disso, a expressão de genes relacionados à atividade da enzima é regulada pela concentração interna de $\mathrm{P}$, sendo maior em plantas deficientes no nutriente (Baldwin et al., 2001).

\section{CONCLUSÕES}

1. Os porta-enxertos cítricos apresentaram resposta diferencial de crescimento e nutrição fosfatada em função do suprimento de $\mathrm{P}$ na solução nutritiva. A eficiência de absorção de $\mathrm{P}$ e, principalmente, de utilização do nutriente para a produção de biomassa também foi variável entre os PEs, indicando diferenças na adaptação à baixa disponibilidade de $\mathrm{P}$.

2. Exceto para o 'Swingle', o baixo suprimento de $\mathrm{P}$ na solução nutritiva elevou a atividade da fosfatase ácida nas raízes dos porta-enxertos, sugerindo capacidade para aproveitamento do $\mathrm{P}$ orgânico em condições de deficiência do nutriente.

3. O sistema radicular do 'Cravo' com EAP e atividade da fosfatase ácida nas raízes igual ou mais elevada do que a dos demais $\mathrm{PE}$, crescimento mais vigoroso e conversão mais eficiente do $\mathrm{P}$ em biomassa indicaram que é o porta-enxerto mais adaptado para solos com baixos teores disponíveis de $\mathrm{P}$.

\section{AGRADECIMENTOS}

À FAPESP pelo apoio financeiro (processo 2007/04634-3); Horticitrus pelo fornecimento dos porta-enxertos cítricos.

\section{LITERATURA CITADA}

ASMAR, F. Variation in activity of root extracellular phytase between genotypes of barley. Plant Soil, 195:61-64, 1997.

BALDWIN, J.C.; KARTHIKEYAN, A.S. \& RAGHOTHAMA, K.G. LEPSE2, a phosphorus starvation-induced novel acid phosphatase from tomato. Plant Physiol., 125:728737, 2001.

BALIGAR, V.C.; FAGÉRIA, N.K. \& HE, Z.L. Nutrient use efficiency in plants. Comm. Soil Sci. Plant Anal., 37:921950, 2001.

BARBER, S.A.; WALKER, J.M. \& VASEY, E.H. Mechanisms for the movement of plant nutrients from the soil and fertilizer to the plant root. Agric. Food Chem., 11:204-207, 1963.

BATAGLIA, O.C.; FURLANI, P.R.; FERRAREZI, R.S. \& MEDINA, C.L. Padrão nutricional de mudas de citros. Araraquara, Vivecitrus/Conplant, 2008.

BATAGLIA, O.C.; FURLANI, A.M.C.; TEIXEIRA, J.P.F.; FURLANI, P.R. \& GALLO, J.R. Método de análise química de plantas Campinas, Instituto Agronômico de Campinas, 1983. (Boletim Técnico, 78)

BATES, T.R. \& LYNCH, J.P. The efficiency of Arabidopsis thaliana (Brassicaceae) root hairs in phosphorus acquisition. Am. J. Bot., 87:964-970, 2000

BESFORD, R.T. A rapid tissue test for diagnosing phosphorus deficiency in the tomato plant. Ann. Bot., 45:225-227, 1980.

BESFORD, R.T. Nutrient imbalances in tomato plants and acid phosphatase activity in the leaves. J. Sci. Food Agric., 30:275-280, 1979. 
BIELESKI, R.L. Phosphate pools, phosphate transport and phosphate availability. Ann Rev. Plant Physiol., 24:225$252,1973$.

CARVALHO, S.A. Produção de porta-enxertos cítricos, sob doses crescentes de nitrato de potássio. Pesq. Agropec. Bras., 29:87-90, 1994.

CHIERA, J.; THOMAS, J. \& RUFTY, T. Leaf initiation and development in soybean under phosphorus stress. J. Exper. Bot., 53:473-481, 2002.

COELHO, P. Purification and characterization of secreted acid phosphatase in phosphorus-deficient Arabidopsis thaliana. Physiol. Plant., 116:293-298, 2002.

DALAL, R.C. Organic phosphorus. Adv. Agron., 29:83-117, 1978.

DARRAH, P.R. The rhizosphere and plant nutrition: A quantitative approach. Plant Soil, 155/156:1-20, 1993.

DREW, M.C. Comparison of the effects of a localized supply of phosphate, nitrate, ammonium and potassium on the root growth of the seminal root system, and the shoot in barley. New Phytol., 75:479-490, 1975.

DUFF, S.M.G.; PLAXTON, W.C. \& LAFEBVRE, D.D. Phosphatestarvation response in plant cells: de novo synthesis and degradation of acid phosphatases. Proc. Nat. Acad. Sci., 88:9538-9542, 1991.

EISSENSTAT, D.M. \& ACHOR, D.S. Anatomical characteristics of roots of citrus rootstocks that vary in specific root length. New Phytol., 141:309-321, 1999.

ELLIOTT, G.C. \& LÄUCHLI, A. Evaluation of an acid phosphatase assay for detection of phosphorus deficiency in leaves of maize (Zea mays L.). J. Plant Nutr., 9:1469$1477,1986$.

ELLIOTT, K.J. \& WHITE, A.S. Effects of light, nitrogen, and phosphorus on red pine seedling growth and nutrient use efficiency. For. Sci., 40:47-58, 1994.

ESPOSTI, M.D. \& SIQUEIRA, D.L. Doses de uréia no crescimento de porta-enxerto de citros produzidos em recipientes. R. Bras. Frutic., 26:136-139, 2004.

FAGERIA, N.K.; WRIGHT, R.J. \& BALIGAR, V.C. Rice cultivar evaluation for phosphorus use efficiency. Plant Soil, 111:105-109, 1988.

FERNANDEZ, D.S. \& ASCENCIO, J. Acid phosphatase activity in bean and cowpea plants grown under phosphorus stress. J. Plant Nutr., 17:229-241, 1994.

FREDEEN, A.; RAO, I.M. \& TERRY, N. Influence of phosphorus nutrition on growth and carbon partitioning in Glycine max. Plant Physiol., 89:335-230, 1989.

FURLANI, A.M.; FURLANI, P.R.; TANAKA, R.T.; MASCARENHAS, H.A.A. \& DELGADO, M.D.P. Variability of soybean germplasm in relation to phosphorus uptake and use efficiency. Sci. Agric., 59:529-536, 2002.

FURLANI, A.M.C.; CLARK, R.B.; MARANVILLE, J.W. \& ROSS, W.M. Root phosphatase activity of sorghum genotypes grown with organic and inorganic sources of phosphorus. J. Plant Nutr., 7:1583-1595, 1984.
FURLANI, P.R.; SILVEIRA, L.C.P.; BOLONHEZI, D. \& FAQUIN, V. Cultivo hidropônico de plantas. Campinas, Instituto Agronômico de Campinas, 1999. (Boletim Técnico, 180)

FURTINI NETO, A.E.; BARROS, N.F.; NOVAIS, R.F. \& OLIVEIRA, M.F.G. Frações fosfatadas em mudas de Eucalyptus. R. Bras. Ci. Solo, 22:267-274, 1998.

GEORGIOU, A. Performance of 'Nova' mandarin on eleven rootstocks in Cyprus. Sci. Hortic., 84:115-126, 2000.

GILBERT, G.S.; KNIGHT, J.D.; VANCE, C.P. \& ALLAN, D.L. Acid phosphatase activity in phosphorus-deficient white lupin roots. Plant Cell Environ., 22:801-810, 1999.

GOURLEY, C.J.P.; ALLAN, D.L. \& RUSSELLE, M.P. Defining phosphorus efficiency in plants. Plant Soil, 155/156:289$292,1993$.

HART, A.L. \& JESSOP, D. Phosphorus fractions in trifoliate leaves of white clover and lotus at various levels of phosphorus supply. New Zealand J. Agric. Res., 26:357$361,1983$.

HELAL, H.M. Varietal differences in root phosphatase activity as related to the utilization of organic phosphates. Plant Soil, 123:161-163, 1990.

HINSINGER, P. Bioavailability of soil inorganic $\mathrm{P}$ in the rhizosphere as affected by root-induced chemical changes: A review. Plant Soil, 237:173-195, 2001.

LIM, J.H.; CHUNG, I.; RYU, S.S.; PARK, M.R. \& YUN, S.J. Differential responses of rice acid phosphatase activities and isoforms to phosphorus deprivation. J. Biochem. Molec. Biol., 36:597-602, 2003

LIU, C.; UMESH, S.M.; UTHAPPA, M.; KONONOWICZ, A.K. \& RAGHOTHAMA, K.G. Tomato phosphate transporter genes are differentially regulated in plant tissues by phosphorus. Plant Physiol., 116:91-99, 1998.

LÓPEZ-CANTERO, I.; RUIZ, J.M. \& HERNADEZ ROMERO, L. Phosphorus metabolism and yield response to increase in nitrogen-phosphorus fertilization: Improvement in greenhouse cultivation of eggplant (Solanum melongena cv. Bonica). J. Agric. Food Chem., 46:1603-1608, 1998.

LYNCH, J.; LAUCHLI, A. \& EPSTEIN, E. Vegetative growth of the common bean in response to phosphorus nutrition. Crop Sci., 31:380-387, 1991.

MALAVOLTA, E.; VITTI, G.C. \& OLIVEIRA, S.A. Avaliação do estado nutricional das plantas: Princípios e aplicações. Piracicaba, Potafos, 1997.

MARSCHNER, H. Mineral nutrition of higher plants. London, Academic Press, 1995. 889p.

MARSCHNER, H. Role of root growth, arbuscular mycorrhiza, and root exudates for the efficiency in nutrient solution. Field Crops Res., 56:203-207, 1998.

MARTINEZ, H.E.P.; NOVAIS, R.F.; RODRIGUES, L.A. \& SACRAMENTO, L.V.S. Phosphate forms in plant and their internal buffering in five soybean cultivars. R. Bras. Ci. Solo, 29:249-257, 2005. 
MATTOS JR., D.; QUAGGIO, J.A.; CANTARELLA, H.; ALVA, A.K. \& GRAETZ, D.A. Response of young citrus trees on selected rootstocks to nitrogen, phosphorus, and potassium fertilization. J. Plant Nutr., 29:1371-1385, 2006.

MOLLIER, A. \& PELLERIN, S. Maize root system growth and development as influenced by phosphorus deficiency. J. Exper. Bot., 50:487-497, 1999.

MORAIS, E.J.; BARROS, N.F.; NOVAIS, R.F. \& BRANDI, R.M. Biomassa e eficiência nutricional de espécies de eucalipto em duas regiões bioclimáticas de Minas de Gerais. R. Bras. Ci. Solo, 14:353-362, 1990.

MOURA, W.M.; LIMA, P.C.; CASALI, V.W.D.; PEREIRA, P.R.G. \& CRUZ, C.D. Eficiência nutricional para fósforo em linhagens de pimentão. Hortic. Bras., 19:174-180, 2001.

NANAMORI, M.; SHINANO, T.; WASAKI, J.; YAMAMURA, T.; RAO, I.M. \& OSAKI, M. Low phosphorus tolerance mechanisms: Phosphorus recycling and photosynthate partitioning in the tropical forage grass, Bachiaria hybrid cultivar mulato compared with rice. Plant Cell Physiol., 45:460-469, 2004.

NIELSEN, K.L.; ESHEL, A. \& LYNCH, J.P. The effect of phosphorus availability on the carbon economy of contrasting common bean (Phaseolus vulgaris L.) genotypes. J. Exper. Bot., 52:329-339, 2001.

O'CONNELL, A.M. \& GROVE, T.S. Acid phosphatase activity karri (Eucalyptus diversicolor F. Muell.) in relation to soil phosphate and nitrogen supply. J. Exper. Bot., 36:13591372,1985

PEREIRA, W.E.; SIQUEIRA, D.L.; PUIATTI, M.; MARTÍNEZ, C.A.; SALOMÃO, L.C.C. \& CECON, P.R. Growth of citrus rootstock under aluminium stress in hydroponic. Sci. Agric., 60:31-41, 2003

PESTANA, M.; VARENNES, A.; ABADIA, J. \& FARIA, E.A. Differential tolerance to iron deficiency of citrus rootstocks grown in nutrient solution. Sci. Hortic., 104:25-36, 2005.

PINTO, C.S.I.; FURTINI NETO, A.E.; NEVES, J.C.L.; FAQUIN, V. \& MORETTI, B.S. Eficiência nutricional de clones de eucalipto na fase de mudas cultivados em solução nutritiva. R. Bras. Ci. Solo, 35:523-533, 2011.

POMPEU JR., J. Porta-enxertos. In: MATTOS JR., D.; DE NEGRI, J.D.; PIO, R.M. \& POMPEU, JR., J., eds. Citros. Campinas, Instituto Agronômico e Fundag, 2005.

QUAGGIO, J.A.; CANTARELLA, H. \& RAIJ, B. van. Phosphorus and potassium soil test and nitrogen leaf analysis as a base for citrus fertilization. Nutr. Cycl. Agroec., 52:67-74, 1998.

QUAGGIO, J.A.; MATTOS JR., D. \& CANTARELLA, H. Fruit yield and quality of sweet orange affected by nitrogen, phosphorus and potassium fertilization in tropical soil. Fruits, 61:293-302, 2006.

QUAGGIO, J.A.; MATTOS JR., D. \& CANTARELLA, H. Portaenxertos. In: MATTOS JR., D.; DE NEGRI, J.D.; PIO, R.M. \& POMPEU JR., J., eds. Citros. Campinas, Instituto Agronômico e Fundag, 2005.
QUISEL, J.D.; WYDOFF, D.D. \& GROSSMAN, A.R. Biochemical characterization of the extracellular phosphatase produced by phosphorus-deprived Chlamydomonas reinhardtii. Plant Physiol., 111:839-848, 1996.

RAO, I.M. \& TERRY, N. Leaf phosphate status, photosynthesis, and carbon partitioning in sugar beet. Plant Physiol. 107:1313-1321, 1995

RICHARDSON, A.E.; HADOBAS, P.A. \& HAYES, J.E. Acid phosphomonoesterase and phytase activities of wheat (Triticum aestivum L.) roots and utilization of organic phosphorus substrates by seedlings grown in sterile culture. Plant Cell Environ., 23:397-405, 2000.

RUBAEK, G.H. \& SIBBESEN, E. Soil phosphorus dynamics in a long-term field experiment at Askov. Biol. Fert. Soil, 20:86-92, 1995

RUIZ, D.; MARTINEZ, V. \& CERDÁ, A. Demarcating specific ion $\left(\mathrm{NaCl}, \mathrm{Cl}^{-}, \mathrm{Na}^{+}\right)$and osmotic effects in the response of two citrus rootstocks to salinity. Sci. Hortic., 80:213-224, 1999.

RUIZ, D.; MARTINEZ, V. \& CERDÁ, A. Citrus response to salinity: Growth and nutrient uptake. Tree Physiol., 17:141150,1997

SANGINGA, N.; LYASSE, O. \& SINGH, B.B. Phosphorus use efficiency and nitrogen balance of cowpea breeding lines in a low $\mathrm{P}$ soil of the derived savanna zone in West Africa. Plant Soil, 220:119-128, 2000.

SARRUGE, J.R. Soluções nutritivas. Summa Phytopatol., 1:231233,1975 .

SHENOY, V.V. \& KALAGUDI, G.M. Enhancing plant phosphorus use efficiency for sustainable cropping. Biotechem. Adv., 23:501-513, 2005.

SIDDIQI, M.Y. \& GLASS, A.D.M. Utilization index: A modified approach to the estimation and comparison of nutrient utilization efficiency in plants. J. Plant Nutr., 4:289-302, 1981.

SINCLAIR, T.R. \& VADEZ, V. Physiological traits for crop yield improvement in low $\mathrm{N}$ and $\mathrm{P}$ environments. Plant Soil, $245: 1-45,2002$

SOBRAL, L.F.; SOUZA, L.F.S.; MAGALHÃES, A.F.J.; SILVA, J.U.B.B. \& LEAL, M.L.S. Respostas da laranjeira-pêra à adubação com nitrogênio, fósforo e potássio em um Latossolo Amarelo dos tabuleiros costeiros. Pesq. Agropec. Bras., 35:307-312, 2000.

SORGONÁ, A.; ABENAVOLI, M.R. \& CACCO, R. A comparative study between two citrus rootstocks: Effect of nitrate on the root morpho-topology and net nitrate uptake. Plant Soil, 270:257-267, 2005.

SPEIR, T.W. \& COWLING, J.C. Phosphatase activities of pastures plants and soils: Relationship with plant productivity and soil $\mathrm{P}$ fertility indices. Biol. Fert. Soils, 12:189-194, 1991.

SWIADER, J.M.; CHYAN, Y. \& FREIJI, F.G. Genotypic differences in nitrate uptake and utilization efficiency in pumpkin hybrids. J. Plant Nutr., 17:1687-1699, 1994. 
SYVERTSEN, J.P. \& GRAHAM, J.H. Phosphorus supply and arbuscular mycorrhizae increase growth and net gas exchange responses of two Citrus spp. grown at elevated $\left[\mathrm{CO}_{2}\right]$. Plant Soil, 208:209-219, 1999.

SYVERTSEN, J.P. \& GRAHAM, J.H. Hydraulic conductivity of roots, mineral nutrition, and leaf gas exchange of citrus rootstocks. J. Am. Soc. Hortic. Sci., 110:865-869, 1985.

THEODORU, M.E. \& PLAXTON, W.C. Metabolic adaptations of plant respiration to nutritional phosphate deprivation. Plant Physiol., 101:339-344, 1993.

TOMSCHA, J.L.; TRULL, M.C.; DEIKMAN, J.; LYNCH, J.P. \& GUILTINAN, M.J. Phosphatase under-producer mutants have altered phosphorus relations. Plant Physiol., 135:334$345,2004$.

VANCE, C.P.; UHDE-STONE, C. \& ALLAN, D.L. Phosphorus acquisition and use: Critical adaptations by plants for securing a nonrenewable resource. New Phytol., 157:423$447,2003$.

YAN, X.; LIAO, H.; TRULL, M.C.; BEEBE, S.E. \& LYNCH, P. Induction of a major leaf acid phosphatase does not confer adaptation to low phosphorus availability in common bean. Plant Physiol., 125:1901-1911, 2001.
YUN, S.J. \& KAEPLER, S.M. Induction of maize acid phosphatase activities under phosphorus starvation. Plant Soil, 237:109-115, 2001.

ZAMBROSI, F.C.B.; ALLEONI, L.F.A. \& CAIRES, E.F. Liming and ionic speciation of an Oxisol under no-till system. Sci. Agric., 62:190-203, 2008.

ZAMBROSI, F.C.B.; MATTOS JR., D. \& SYVERTSEN, J.P. Plant growth, leaf photosynthesis, and nutrient-use efficiency of citrus rootstocks decrease with phosphite supply. J. Plant Nutr. Soil Sci., 174:487-495, 2011.

ZAMBROSI, F.C.B.; MATTOS Jr., D.; BOARETTO, R.M.; QUAGGIO, J.A. \& MURAOKA, T.; SYVERTSEN, J.P. Contribution of phosphorus absorption and remobilization for citrus growth. Plant Soil, 2012b. No prelo.

ZHANG, H.; HUANG, Y.; YE, X.; SHI, L. \& XU, F. Genotypic differences in phosphorus acquisition and the rhizosphere properties of Brassica napus in response to low phosphorus stress. Plant Soil, 320:91-102, 2009.

WANG, X.; SHEN, J. \& LIAO, H. Acquisition or utilization, which is more critical for enhancing phosphorus efficiency in modern crops? Plant Sci., 179:302-306, 2010. 